\title{
Laser-induced dissociative recombination of carbon dioxide
}

\author{
Hongtao Hu $\odot,{ }^{1}$ Seyedreza Larimian, ${ }^{1}$ Sonia Erattupuzha, ${ }^{1}$ Jin Wen $\odot,{ }^{2,3, *}$ Andrius Baltuška, ${ }^{1}$ \\ Markus Kitzler-Zeiler, ${ }^{1}$ and Xinhua Xie (谢新华) ${ }^{1,4, \dagger}$ \\ ${ }^{1}$ Photonics Institute, Technische Universität Wien, A-1040 Vienna, Austria \\ ${ }^{2}$ Institute of Organic Chemistry and Biochemistry, AS CR, 16610 Praha 6, Czech Republic \\ ${ }^{3}$ Institute of Theoretical Chemistry, University of Vienna, A-1090 Vienna, Austria \\ ${ }^{4}$ SwissFEL, Paul Scherrer Institute, 5232 Villigen PSI, Switzerland
}

(Received 22 June 2019; revised manuscript received 20 September 2019; published 5 December 2019)

\begin{abstract}
We experimentally investigate laser-induced dissociative recombination of $\mathrm{CO}_{2}$ in linearly polarized strong laser fields with coincidence measurements. Our results show laser-induced dissociative processes with electron recombination after laser-induced double ionization. After double ionization, one electron can recombine to one of the two ionic fragments during laser-induced molecular dissociation. Our measurements reveal that the recombination probability of the second ionized electron is three times as high as that of the first ionized electron.
\end{abstract}

DOI: 10.1103/PhysRevResearch.1.033152

Dissociative recombination refers to the process where a positively charged molecular ion captures a free electron, upon which a (highly) excited molecular complex is formed that subsequently dissociates into fragments $[1,2]$. It is one of the most important processes in plasmas, for instance in planetary atmospheres, fusion plasmas, and laser physics [3,4]. Most of experimental studies on dissociative recombination were performed at large facilities, such as heavy-ion storage rings in which the molecular ions are produced by electronimpact ionization [1,2].

In the past decades, femtosecond lasers became a versatile tool to reveal nuclear and electron dynamics of atoms and molecules [5-8], due to their ultrashort pulse durations and high peak intensities. When exposed to a strong laser field, molecules can become ionized or excited, which may cause further molecular reactions, such as dissociation and isomerization. Laser-induced dissociative recombination (LIDR) is one of such reactions, which has attracted the interest of researchers for a decade [9]. In the literature on strong-field laser science this process is also referred to dissociative frustrated ionization [10], where electrons are released from a molecule by the strong laser field and afterwards an electron recombines with one of the ionic fragments during the dissociation of the molecule. To the best of our knowledge, the research on LIDR only focused on homonuclear diatomic molecules, such as $\mathrm{H}_{2}$, $\mathrm{D}_{2}$, and $\mathrm{Ar}_{2}$ [11-14].

In this work, we investigated the LIDR of a triatomic molecule $\mathrm{CO}_{2}$ in linearly polarized laser fields with a reaction microscope. In comparison with a diatomic, the complexity

\footnotetext{
*Corresponding author: jin.wen@univie.ac.at

${ }^{\dagger}$ Corresponding author: xinhua.xie@tuwien.ac.at

Published by the American Physical Society under the terms of the Creative Commons Attribution 4.0 International license. Further distribution of this work must maintain attribution to the author(s) and the published article's title, journal citation, and DOI.
}

of a triatomic molecule is significantly higher due to the increasing of molecular motion modes and electron configurations [15]. Dissociative recombination of $\mathrm{CO}_{2}$ is expected to play an important role in the interaction of $\mathrm{CO}_{2}$ with photons and electrons [16]. It is valuable to gain knowledge on dissociative recombination of $\mathrm{CO}_{2}$ for further understanding of laser-induced reaction in molecular complex. In this paper, we are trying to understand the dynamics of the LIDR of $\mathrm{CO}_{2}$ through answering the following two questions: (1) how does the LIDR happen in $\mathrm{CO}_{2}$ and (2) which of the two released electrons contributes more to the recombination in the LIDR process?

In the experiment, coincidence measurements of threedimensional momenta of resulting particles are achieved using a reaction microscope [17]. A schematics of the coincidence measurement of the LIDR process is shown in Fig. 1(a). Charged particles, e.g., $\mathrm{CO}^{+}$and electrons, are guided to the multihit position- and time-sensitive detectors by a homogeneous dc field of $10.5 \mathrm{~V} / \mathrm{cm}$ and a uniform magnetic field of $12 \mathrm{G}$. Excited neutral particles, e.g., O*, with an initial momentum vector pointing towards the detector are registered with a detection acceptance angle of about $70^{\circ}[9,11,18,19]$. In the off-line data analysis, momentum conservation conditions between detected particles are applied to minimize background signals. In the measurements, linearly polarized 25-fs, 790-nm laser pulses (along $z$ direction) are delivered from a Ti:sapphire laser amplifier with a repetition rate of $5 \mathrm{kHz}$. The laser beam is focused onto the $\mathrm{CO}_{2}$ gas jet in the interaction chamber with a spherical mirror which has a focal length of $60 \mathrm{~mm}$. $\mathrm{CO}_{2}$ molecules are introduced to the interaction chamber from a supersonic gas jet system which consists of a gas nozzle followed by a skimmer. The laser peak intensity at the focus is about $5 \times 10^{14} \mathrm{~W} / \mathrm{cm}^{2}$, so that $\mathrm{CO}_{2}$ molecules can be singly or doubly ionized and subsequently dissociate into fragments. More details about the experimental setup can be found in our previous publications [20-24].

We now turn to the question how the LIDR happens in $\mathrm{CO}_{2}$. The two possible LIDR processes can be written 

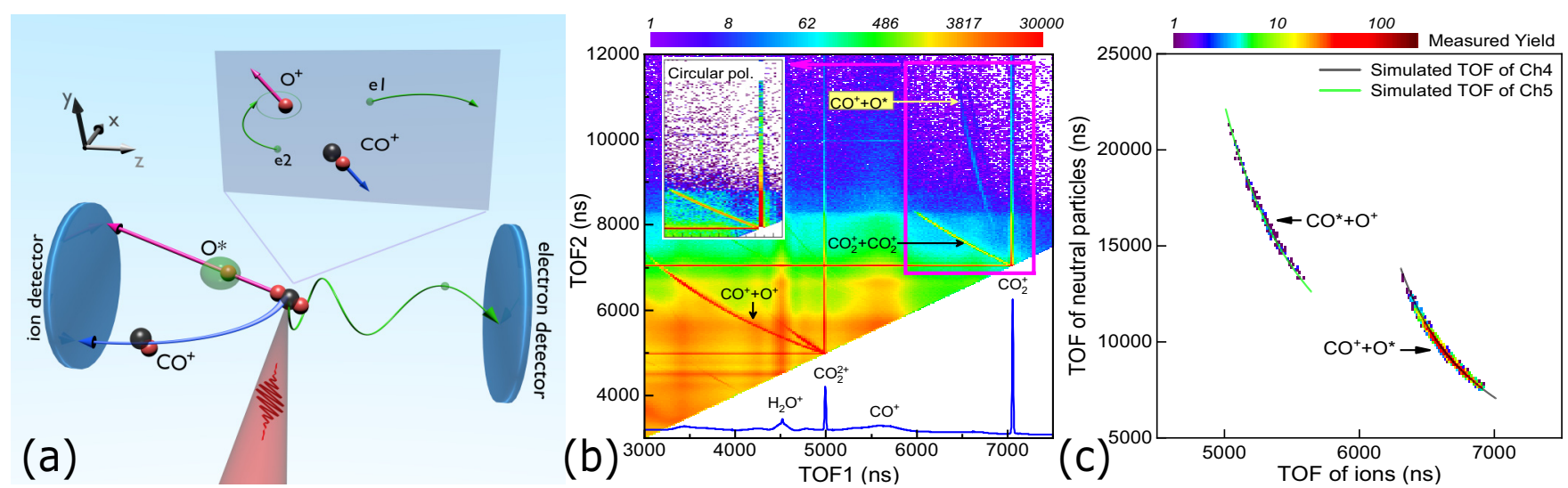

FIG. 1. (a) LIDR of $\mathrm{CO}_{2}$ induced by the intense laser field, and the coincidence detection of resulting particles with a reaction microscope. (b) Measured PIPICO distribution of $\mathrm{CO}_{2}$ in linearly polarized laser field. The blue curve at the bottom of (b) is the time-of-flight spectrum of the experiment. The insert of (b) shows the PIPICO distribution for the measurement with circular polarized laser pulses in the range marked by the pink rectangle. (c) Coincidence selection of LIDR processes in PIPICO distribution with corresponding simulated PIPICO lines.

as $\mathrm{CO}_{2} \rightarrow \mathrm{CO}^{+}+\mathrm{O}^{*}+e(\mathrm{Ch} 4)$ or $\mathrm{CO}_{2} \rightarrow \mathrm{CO}^{*}+\mathrm{O}^{+}+e(\mathrm{Ch} 5)$ (see Table I for channel definitions). With the reaction microscope, we achieve complete coincidence detection of the three particles (an ion, a neutral, and an electron) generated in these LIDR processes. Figure 1(b) shows the measured photoion photoion coincidence (PIPICO) distribution. The sharp parabolic PIPICO lines are signals of two-body dissociation channels as indicated in the figure, among which the LIDR channel $\mathrm{Ch} 4$ can be clearly identified. The weak LIDR channel Ch5 is not visible on the intensity scale of Fig. 1(b). However, after applying coincidence selection on the momentum sums of the two particles $\left(\left|p 1_{x}+p 2_{x}\right|<4\right.$ a.u., $\left|p 1_{y}+p 2_{y}\right|<8$ a.u. and $\left|p 1_{z}+p 2_{z}\right|<3$ a.u.), the two LIDR processes can be selected out from the PIPICO distribution, as shown in Fig. 1(c). The simulated PIPICO lines in Fig. 1(c) represent for the corresponding processes, which confirms the observation of the two LIDR processes. The branching ratios of related processes are summarized in Table I. In the following, we will focus on $\mathrm{Ch} 4$ first.

As shown in the inset of Fig. 1(b), no LIDR signals are detected with circularly polarized light. This observation contradicts a previous experimental observation of hydrogen [25], in which Rydberg states are formed via resonant multiphoton excitation by irradiation of the circularly polarized laser fields. The observation of no LIDR signals with circular pulses con-

TABLE I. The branching ratios of resulting channels from $\mathrm{CO}_{2}$ in strong laser fields. The peak intensity of the circular pulse was $2.5 \times 10^{14} \mathrm{~W} / \mathrm{cm}^{2}$.

\begin{tabular}{llcc}
\hline \hline & \multirow{2}{*}{$\begin{array}{c}\text { Dynamics } \\
\text { Channels }\end{array}$} & \multicolumn{2}{c}{ Branching ratio } \\
\cline { 3 - 4 } & $\left(\mathrm{CO}_{2} \rightarrow\right)$ & Linear & Circular \\
\hline Ch1 & $\mathrm{CO}_{2}^{+}+e$ & $24.52 \%$ & $46.38 \%$ \\
Ch2 & $\mathrm{CO}_{2}^{2+}+2 e$ & $14.10 \%$ & $1.60 \%$ \\
Ch3 & $\mathrm{CO}^{+}+\mathrm{O}^{+}+2 e$ & $8.01 \%$ & $5.62 \%$ \\
Ch4 & $\mathrm{CO}^{+}+\mathrm{O}^{*}+e$ & $0.10 \%$ & - \\
Ch5 & $\mathrm{CO}^{*}+\mathrm{O}^{+}+e$ & $0.002 \%$ & - \\
Ch6 & $\mathrm{CO}^{+}+\mathrm{O}^{+}+e$ & $16.38 \%$ & $12.65 \%$ \\
Ch7 & $\mathrm{CO}^{+}+\mathrm{O}+e$ & $36.87 \%$ & $33.75 \%$ \\
\hline \hline
\end{tabular}

firms that Rydberg states are formed through frustrated double ionization in this work, which is a rescattering-like process $[9,10,23,24,26]$. This process will be strongly suppressed by circularly polarized laser fields [6,27-29].

To gain insight into the quantum states involved in our observations of laser-induced dissociation of $\mathrm{CO}_{2}$, we performed quantum chemical simulations to obtain the potential energy curves of the relevant states. Multiconfiguration complete-active-space self-consistent field theory (CASSCF) should be employed for an accurate description of the electronic structure of $\mathrm{CO}_{2}$ in excited states. To simulate the dissociation processes of $\mathrm{CO}_{2}, \mathrm{CO}_{2}^{+}$, and $\mathrm{CO}_{2}^{2+}$ along the $\mathrm{C}-\mathrm{O}$ stretching coordinates in highly-excited states, a multistate complete active space perturbation theory of second order (MS-CASPT2) [30], which further considered dynamic correlations, was carried out using Molcas 8.2 [31]. In the active spaces of CASSCF calculations, 10, 9, and 8 electrons in 15 orbitals are included in $\mathrm{CO}_{2}, \mathrm{CO}_{2}^{+}$, and $\mathrm{CO}_{2}^{2+}$ molecules respectively, when they were all kept in $\mathrm{C}_{2} v$ symmetry. In searching for potential energy curves along $\mathrm{C}-\mathrm{O}$ bonds of $\mathrm{CO}_{2}^{+}$, the state-averaged CASSCF wave functions for 20 singlet excited states were used in MS-CASPT2 calculations with ANO-RCC-VTZP basis set, while O-C-O valence angles were constrained to $180^{\circ}$. The calculated potential energy curves (PECs) of $\mathrm{CO}_{2}, \mathrm{CO}_{2}^{+}$, and $\mathrm{CO}_{2}^{2+}$ along the $\mathrm{C}-\mathrm{O}$ bonds are presented in Fig. 2.

When $\mathrm{CO}_{2}$ interacts with a strong laser field, single or double ionization may occur by removing of one or two valence electrons. Since the energy gaps between different molecular orbitals (MOs) are rather small, direct removal of electrons from a low-lying MO is possible, resulting in electronically excited states [32,33]. As shown in Fig. 2, removal of one or two electrons from the HOMO of $\mathrm{CO}_{2}$ leads to stable ground states of the $\mathrm{CO}_{2}^{+}$or $\mathrm{CO}_{2}^{2+}$, observed as $\mathrm{Ch} 1$ or $\mathrm{Ch} 2$. On the other hand, dissociative electronically excited states of cation or dication can be reached through removal of at least one electron from low-lying MOs, yielding Ch3, Ch6, and $\mathrm{Ch} 7$. In the strong field interaction, high-lying Rydberg states can be populated through the so-called frustrated field ionization [9,10,23,26]. In case of molecules, such Rydberg states can be dissociative and close to the ionization threshold 


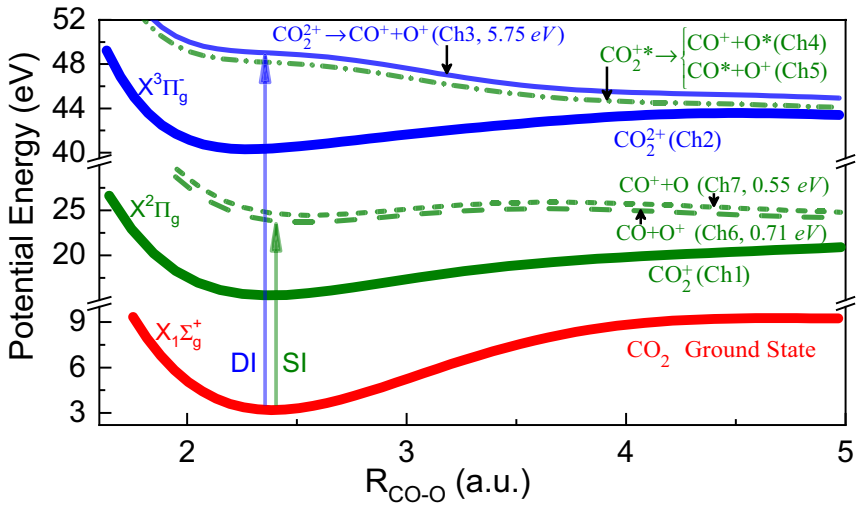

FIG. 2. Simulated potential energy curves (PECs) of $\mathrm{CO}_{2}, \mathrm{CO}_{2}^{+}$, and $\mathrm{CO}_{2}^{2+}$ over one $\mathrm{C}-\mathrm{O}$ stretching coordinate. The bold curves are the ground states of $\mathrm{CO}_{2}, \mathrm{CO}_{2}^{+}$, and $\mathrm{CO}_{2}^{2+}$. The dash-dotted curve is a schematic for a high-lying Rydberg state of $\mathrm{CO}_{2}^{+}$which is directly below the dissociative state of $\mathrm{CO}_{2}^{2+}$ (solid blue curve). The simulated KERs of dissociation channels are included. (SI: single ionization; DI: double ionization.)

to a higher charged state, schematically shown in Fig. 2, where a high-lying Rydberg state of $\mathrm{CO}_{2}^{+}$lies very close to one PEC of an electronically excited state of $\mathrm{CO}_{2}^{2+}$. The kinetic energy release (KER) of the dissociation from these two states is similar because of negligible influence of the Rydberg electron to the dissociation process.

Since the measured momentum/energy distributions of detected particles contain information on the nuclear dynamics during strong field interaction, we first checked measured momentum and KER distributions of dissociation channels. The momentum distributions of the fragments generated through $\mathrm{Ch} 3$ and $\mathrm{Ch} 4$ are plotted in Figs. 3(a) and 3(b). Except for different acceptance angles, the momentum distributions of both channels are similar and peak along the laser polarization direction. Such anisotropic distributions indicate electron removal from low-lying $\sigma$-type MOs during the strong field ionization [20,21,32]. It implies that $\mathrm{Ch} 3$ and $\mathrm{Ch} 4$ originate from electronically excited states formed by removal an electron from lower-lying MOs, as in Fig. 2.

The KER distributions of $\mathrm{Ch} 3$ and $\mathrm{Ch} 4$ are plotted in Fig. 3(c). One clear observation is that the KER distribution of $\mathrm{Ch} 4$ (red squares) is almost the same as that of $\mathrm{Ch} 3$ (blue areas) with a peak at $5.8 \mathrm{eV}$, which agrees well with the simulated KER of $5.75 \mathrm{eV}$. Since the KER distribution is determined by the involved electronic states (see Fig. 2), this observation indicates that the dissociative nuclear wave packets of $\mathrm{Ch} 3$ and $\mathrm{Ch} 4$ evolve on PECs with similar shapes. Furthermore, the same KER distributions of $\mathrm{Ch} 3$ and $\mathrm{Ch} 4$ not only shows that the recombination of the ion and the electron occurs during the molecular dissociation, but also provides a clear evidence that the electron is recaptured into high-lying Rydberg states such that the nuclear charge is not fully shielded by the trapped electron and leading to similar KERs of the two channels.

To summarize the answer to the first question, the LIDR happens as laser-induced dissociative double ionization through the removal of electrons from low-lying MOs
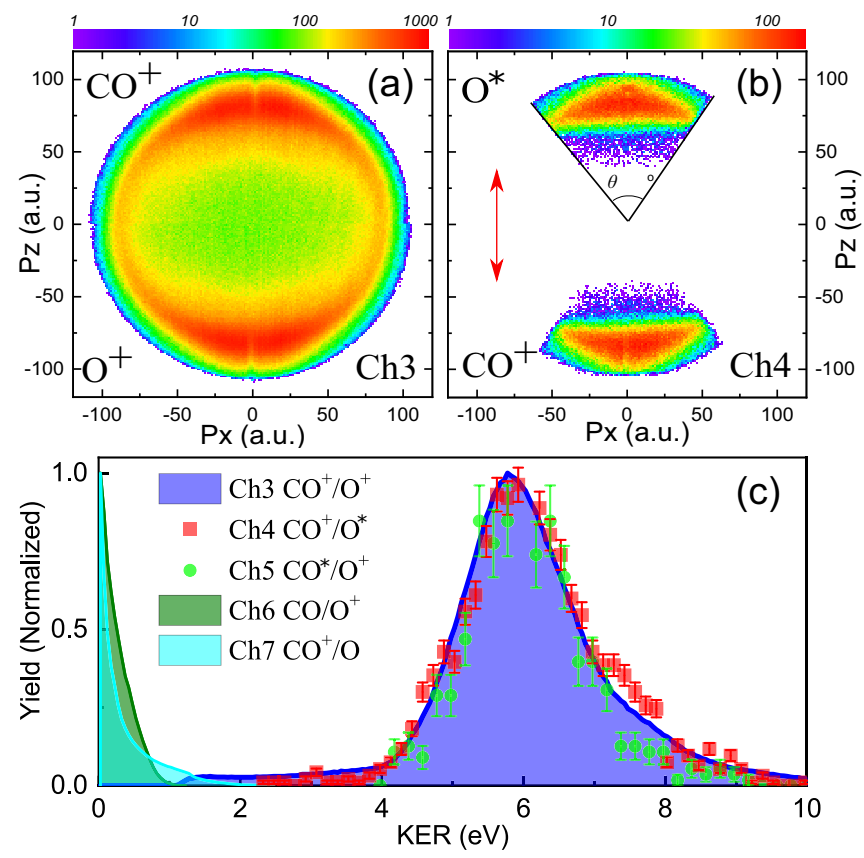

FIG. 3. Measured momentum distributions in the $x-z$ plane for (a) $\mathrm{Ch} 3$ and (b) Ch4. Laser polarized direction is indicated by the red arrow. (c) Normalized KER spectra of different dissociation channels.

followed by the electron recombination to one of the ionic fragments during the dissociation of the molecule.

Now we turn to the second question: which of the released electrons recombines more favorably with the parent ion? To answer it, we analyze momentum distributions of electrons and ions along the laser polarized direction. The final momentum of photoelectrons from the strong field interaction is determined by the vector potential of the laser field at the electron birth time [5,34], therefore electron momentum distributions contain information about the ionization dynamics during strong field interaction. Due to momentum conservation, the electron momenta can be derived from measured ionic momenta. Figure 4 shows the measured ion momentum distributions of $\mathrm{Ch} 1$ to $\mathrm{Ch} 5$. For the dissociative processes the momentum sums of the two fragments are analyzed. First, we compare the momentum distributions of direct ionization processes $(\mathrm{Ch} 1, \mathrm{Ch} 2$, and $\mathrm{Ch} 3)$. At the laser intensity of our measurements, single ionization happens at the leading edge of the laser pulses due to saturation, which leads to a rather narrow momentum distribution. On the other hand, in case of double ionization, we notice that the momentum distribution of the dissociative process $(\mathrm{Ch} 3)$ is broader than that of the nondissociative process (Ch2). At the laser intensity of $5 \times 10^{14} \mathrm{~W} / \mathrm{cm}^{2}$, the dominant double ionization process happens sequentially, which means the two electron are removed one after the other [35]. As shown in Fig. 3(a), the width of the sum momentum distribution for double ionization is broader than that for single ionization. It is due to the broader momentum width of the second electron which is removed at a higher laser intensity. The dissociative process $(\mathrm{Ch} 3)$ originates from electronically excited states of $\mathrm{CO}_{2}^{2+}$, which involves removal of an electron from a lower-lying MO during the first ionization step. As this lower-lying MO exhibits a 


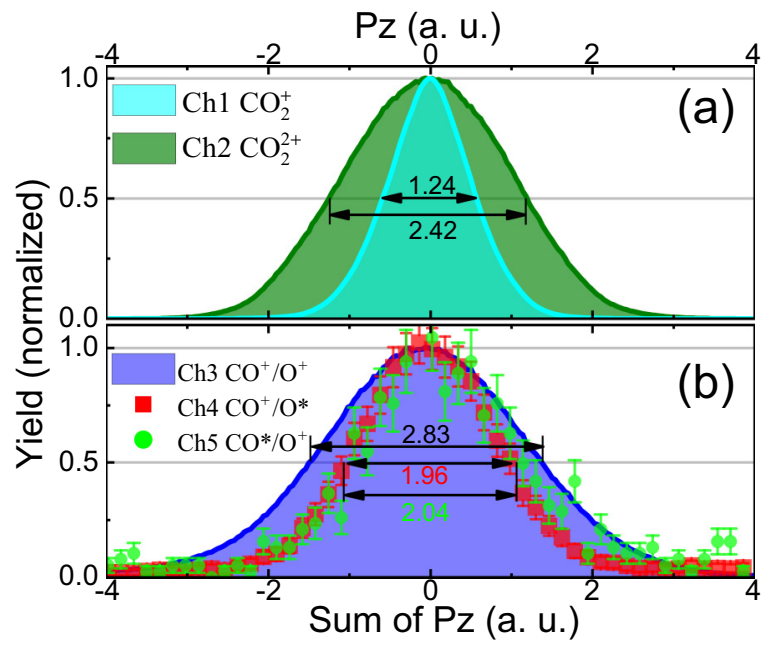

FIG. 4. Measured ion momentum distributions along the laser polarization direction for $\mathrm{Ch} 1$ and $\mathrm{Ch} 2$ (a) and ion momentum sum distribution for $\mathrm{Ch} 3, \mathrm{Ch} 4$, and $\mathrm{Ch} 5$ (b). The black arrows indicate their full width at half maximum. To be noted, the momentum distributions of $\mathrm{Ch} 2$ and $\mathrm{Ch} 4$ contain the information of the two released electrons. The momentum resolution along the laser polarization direction is 0.05 a.u.. The error bars of $\mathrm{Ch} 4$ and $\mathrm{Ch} 5$ represent the statistic error of the measurements.

larger ionization potential than the HOMO [20], dissociative double ionization (Ch3) happens at an effectively higher laser intensity. This leads to a broader momentum distribution than that of nondissociative double ionization. Now, we compare the momentum distributions of $\mathrm{Ch} 3$ and $\mathrm{Ch} 4$. It is clear that the momentum distribution of $\mathrm{Ch} 4$ is much narrower than that of Ch3. For Ch4 only one electron is released, with the recombination of either the first or the second ionized electron.

In the following, we will quantify the contributions of the first and second electrons to the overall recombination yield from the measured momentum widths. Since double ionization happens mainly sequentially in our experiment, it is reasonable to assume that the first ionization step is saturated. We now can use the momentum width for single ionization (Ch1) as that of the first ionization step $\left(w_{e 1}\right)$. Then the momentum width of the second ionization step $\left(w_{e 2}\right)$ can be derived from the sum of measured electron momentum width $\left(w_{e 1+e 2}\right)$ with the relation of $w_{e 1+e 2}=\sqrt{w_{e 1}^{2}+w_{e 2}^{2}}$. From the measured widths of the ion momentum distribution of Ch1 (1.24 a.u.) and Ch2 (2.42 a.u.), we obtain the momentum width of the second ionization step of $\mathrm{Ch} 2$ to be 2.08 a.u.. With the knowledge that the second ionization of $\mathrm{Ch} 3$ is similar to nondissociative double ionization (Ch2) [20], we used the obtained momentum width of the second ionization step of Ch2 to get the momentum width of the first ionization step by removing an electron from low-lying MOs, which yields 1.92 a.u.. Since only one of these two electrons recombines during dissociative double ionization, the measured electron momentum distribution is determined by the recombination probabilities of the two electrons $\left[S_{\mathrm{Ch} 4}=\alpha_{e 2} \exp \left(-4 \log 2 p_{z}^{2} / w_{e 1}^{2}\right)+\right.$ $\left.\alpha_{e 1} \exp \left(-4 \log 2 p_{z}^{2} / w_{e 2}^{2}\right)\right]$. With the obtained widths of $w_{e 1}=$ 1.92 a.u. and $w_{e 2}=2.08$ a.u., we performed a fitting of the electron momentum distribution with $S_{\mathrm{Ch} 4}$ and got the recombination probabilities of the first and second electron: $\alpha_{e 1}=$ $23 \%$ and $\alpha_{e 2}=77 \%$. The result shows that the recombination probability of the second electron is about three times as high as that of the first electron. This observation agrees with previous measurements on $\mathrm{D}_{2}$ [14] and argon atoms [36]. A straightforward explanation could be that the diffusion of the second electron wave packet is much less than the first electron at the conclusion of the laser field and thus it has a higher recapture probability.

In the end, we compare two LIDR processes ( $\mathrm{Ch} 4$ and Ch5). Both the KER spectrum [Fig. 3(c)] and the ion sum momentum distribution [Fig. 4(b)] along the laser polarization of $\mathrm{Ch} 5$ is similar to that of $\mathrm{Ch} 4$, which strongly implies that the two channels undergo similar nuclear and electron dynamics. To compare with $\mathrm{Ch} 4$, the only difference in $\mathrm{Ch} 5$ is that during the dissociation one electron recombines to the $\mathrm{CO}^{+}$fragment other than the $\mathrm{O}^{+}$. One clear observation is that the yield of $\mathrm{Ch} 4$ is much higher than that of $\mathrm{Ch} 5$, with a yield ratio of about 50 between electron localized to $\mathrm{O}^{+}$ and $\mathrm{CO}^{+}$, as shown in Table I. A straightforward explanation of this observation can be that the electronegativity of $\mathrm{O}^{+}$ is higher than that of $\mathrm{CO}^{+}$which leads to a higher electron recombination probability to $\mathrm{O}^{+}$. We would like to note that the dissociative recombination process observed in our experiments is differ from those performed in storage rings with $\mathrm{CO}_{2}^{2+}$ in the electronic ground state [2].

In summary, we experimentally investigated the laserinduced dissociative recombination processes of $\mathrm{CO}_{2}$ with coincidence detection of all involved particles. The measured KER and momentum distributions allow for understanding of both nuclear and electron dynamics during the LIDR of $\mathrm{CO}_{2}$. Our measurements clearly show that LIDR of $\mathrm{CO}_{2}$ originates from the recombination of one electron to one of the two ionic fragments during laser-induced dissociative double ionization. An analysis of electron momentum distributions shows that the second emitted electron has a much higher probability to recombine with the ionic fragment during the laser-induced dissociative processes. Based on the findings in this work and previous works on diatomic molecules, we believe that LIDR is a general process in strong field interaction with molecules.

This work was financed by the Austrian Science Fund (FWF) under Grants No. P25615-N27, No. P30465-N27, No. M2675-N30, and No. P28475-N27. J.W. thanks the Institute of Organic Chemistry and Biochemistry, Academy of Sciences of the Czech Republic (RVO: 61388963).
[1] M. Larsson, Annu. Rev. Phys. Chem. 48, 151 (1997).

[2] K. Seiersen, A. Al-Khalili, O. Heber, M. J. Jensen, I. B. Nielsen, H. B. Pedersen, C. P. Safvan, and L. H. Andersen, Phys. Rev. A 68, 022708 (2003).
[3] D. Zajfman, J. Mitchell, D. Schwalm, and B. Rowe, Dissociative Recombination: Theory, Experiment and Applications III (World Scientific, Singapore, 1996).

[4] J. L. Fox and A. B. Hać, Icarus 228, 375 (2014). 
[5] R. Pazourek, S. Nagele, and J. Burgdorfer, Rev. Mod. Phys. 87, 765 (2015).

[6] P. B. Corkum and F. Krausz, Nat. Phys. 3, 381 (2007).

[7] J. H. Posthumus, Rep. Prog. Phys. 67, 623 (2004).

[8] T. Brabec and F. Krausz, Rev. Mod. Phys. 72, 545 (2000).

[9] T. Nubbemeyer, K. Gorling, A. Saenz, U. Eichmann, and W. Sandner, Phys. Rev. Lett. 101, 233001 (2008).

[10] B. Manschwetus, T. Nubbemeyer, K. Gorling, G. Steinmeyer, U. Eichmann, H. Rottke, and W. Sandner, Phys. Rev. Lett. 102, 113002 (2009).

[11] W. Zhang, Z. Yu, X. Gong, J. Wang, P. Lu, H. Li, Q. Song, Q. Ji, K. Lin, J. Ma et al., Phys. Rev. Lett. 119, 253202 (2017).

[12] J. Wu, A. Vredenborg, B. Ulrich, L. P. H. Schmidt, M. Meckel, S. Voss, H. Sann, H. Kim, T. Jahnke, and R. Dorner, Phys. Rev. Lett. 107, 043003 (2011).

[13] J. McKenna, S. Zeng, J. J. Hua, A. M. Sayler, M. Zohrabi, N. G. Johnson, B. Gaire, K. D. Carnes, B. D. Esry, and I. Ben-Itzhak, Phys. Rev. A 84, 043425 (2011).

[14] W. Zhang, H. Li, X. Gong, P. Lu, Q. Song, Q. Ji, K. Lin, J. Ma, H. Li, F. Sun et al., Phys. Rev. A 98, 013419 (2018).

[15] G. Herzberg, Molecular Spectra and Molecular Structure: I. Spectra of Diatomic Molecules, 2nd ed. (Krieger Publishing Company, Malabar, Florida, 1989), Vol. 1; Molecular Spectra and Molecular Structure 2. Infrared and Raman Spectra of Polyatomic Molecules, 2nd ed. (Krieger Publishing Company, Malabar, Florida, 1989), Vol. 1.

[16] N. Vinci, N. d. Ruette, F. O. W. Tamo, O. Motapon, M. Fifirig, O. Crumeyrolle, X. Urbain, J. Tennyson, and I. F. Schneider, J. Phys.: Conf. Ser. 4, 162 (2005).

[17] J. Ullrich, R. Moshammer, A. Dorn, R. Dorner, L. P. H. Schmidt, and H. Schmidt-Bocking, Rep. Prog. Phys. 66, 1463 (2003).

[18] M. Barat, J. C. Brenot, J. A. Fayeton, and Y. J. Picard, Rev. Sci. Instrum. 71, 2050 (2000).

[19] B. Berry, M. Zohrabi, D. Hayes, U. Ablikim, B. Jochim, T. Severt, K. D. Carnes, and I. Ben-Itzhak, Rev. Sci. Instrum. 86, 046103 (2015).

[20] S. Erattupuzha, S. Larimian, A. Baltuška, X. Xie, and M. Kitzler, J. Chem. Phys. 144, 024306 (2016).
[21] S. Larimian, S. Erattupuzha, S. Mai, P. Marquetand, L. González, A. Baltuška, M. Kitzler, and X. Xie, Phys. Rev. A 95, 011404(R) (2017).

[22] X. Xie, T. Wang, S. G. Yu, X. Y. Lai, S. Roither, D. Kartashov, A. Baltuška, X. J. Liu, A. Staudte, and M. Kitzler, Phys. Rev. Lett. 119, 243201 (2017).

[23] S. Larimian, S. Erattupuzha, C. Lemell, S. Yoshida, S. Nagele, R. Maurer, A. Baltuška, J. Burgdörfer, M. Kitzler, and X. Xie, Phys. Rev. A 94, 033401 (2016).

[24] S. Larimian, C. Lemell, V. Stummer, J.-W. Geng, S. Roither, D. Kartashov, L. Zhang, M.-X. Wang, Q. Gong, L.-Y. Peng et al., Phys. Rev. A 96, 021403(R) (2017).

[25] W. Zhang, X. Gong, H. Li, P. Lu, F. Sun, Q. Ji, K. Lin, J. Ma, H. Li, J. Qiang et al., Nat. Commun. 10, 757 (2019).

[26] B. Ulrich, A. Vredenborg, A. Malakzadeh, M. Meckel, K. Cole, M. Smolarski, Z. Chang, T. Jahnke, and R. Dörner, Phys. Rev. A 82, 013412 (2010).

[27] P. B. Corkum, Phys. Rev. Lett. 71, 1994 (1993).

[28] M. Chini, K. Zhao, and Z. H. Chang, Nat. Photon. 8, 178 (2014).

[29] L. Gallmann, C. Cirelli, and U. Keller, Annu. Rev. Phys. Chem. 63, 447 (2012).

[30] J. Finley, P.-k. Malmqvist, B. O. Roos, and L. Serrano-Andrés, Chem. Phys. Lett. 288, 299 (1998).

[31] F. Aquilante, J. Autschbach, R. K. Carlson, L. F. Chibotaru, M. G. Delcey, L. D. Vico, I. F. Galván, N. Ferré, L. M. Frutos, L. Gagliardi et al., J. Comput. Chem. 37, 506 (2016).

[32] X. Xie, K. Doblhoff-Dier, H. Xu, S. Roither, M. S. Schöffler, D. Kartashov, S. Erattupuzha, T. Rathje, G. G. Paulus, K. Yamanouchi et al., Phys. Rev. Lett. 112, 163003 (2014).

[33] X. Xie, S. Roither, M. Schöffler, E. Lötstedt, D. Kartashov, L. Zhang, G. G. Paulus, A. Iwasaki, A. Baltuška, K. Yamanouchi et al., Phys. Rev. X 4, 021005 (2014).

[34] E. Goulielmakis, V. S. Yakovlev, A. L. Cavalieri, M. Uiberacker, V. Pervak, A. Apolonski, R. Kienberger, U. Kleineberg, and F. Krausz, Science 317, 769 (2007).

[35] L. Pei and C. Guo, Phys. Rev. A 82, 021401(R) (2010).

[36] S. Larimian, S. Erattupuzha, A. Baltuška, M. Kitzler, and X. Xie, arXiv:1801.03738v1. 